\title{
MOTIVACIONES PARA EL EJERCICIO FÍSICO Y SU RELACIÓN CON LA SALUD MENTAL Y FÍSICA: UN ANÁLISIS DESDE EL GENERO
}

\author{
Bisquert-Bover, $\mathbf{M}$. \\ Salusex. Dpto. Psicología Básica, Clínica y Psicobiología \\ Universitat Jaume I de Castelló \\ al395816@uji.es \\ Ballester-Arnal, R \\ Salusex. Dpto. Psicología Básica, Clínica y Psicobiología \\ Universitat Jaume I de Castelló \\ Gil-Llario, M.D. \\ Salusex. Dpto. de Psicología Evolutiva y de la Educación \\ Universitat de València, Spain. \\ Elipe-Miravet, $\mathbf{M}$. \\ Salusex. Dpto. Psicología Básica, Clínica y Psicobiología \\ Universitat Jaume I de Castelló \\ López-Fando Galdón, $\mathbf{M}$. \\ Salusex. Dpto. Psicología Básica, Clínica y Psicobiología \\ Universitat Jaume I de Castelló
}

Recepción Artículo: 18 enero 2020 Admisión Evaluación: 4 marzo 2020 Informe Evaluador 1: 13 marzo2020 Informe Evaluador 2: 18 marzo2020 Aprobación Publicación: 20 abril 2020

\section{RESUMEN}

El ejercicio físico es una actividad física planificada y estructurada con un objetivo final, constituyendo una herramienta preventiva de las enfermedades no transmisibles y un factor protector de la salud física y mental. Asimismo, las motivaciones para realizarlo son fundamentales y pueden verse influidas por el género. El objetivo del estudio es analizar las diferentes motivaciones para hacer ejercicio en función del género, así como observar la relación entre la realización de ejercicio físico y la percepción de salud física y mental. Para ello, una muestra compuesta por un total de 600 jóvenes universitarios (50\% hombres y $50 \%$ mujeres), cumplimentó el cuestionario online de Estilos de Vida y Salud (Giménez-García y Ballester-Arnal, 2017). Los análisis mostraron diferencias significativas en la realización habitual de ejercicio entre hombres (61.7\%) y mujeres (45.3\%) (Chi² $=16.01$; $\mathrm{p}$ s.001). Asimismo, se observaron diferencias según género en todas las motivaciones para realizar ejercicio, exceptuando el motivo relativo a "estar más delgado/a" (Chi $\left.{ }^{2}=1.00 ; p=.317\right)$, siendo los hombres los que mostraron mayor prevalencia en todos los casos. En relación con las motivaciones para no realizar ejercicio, se observan diferencias en los motivos relativos a la "falta de tiempo" (Chi $\left.{ }^{2}=7.72 ; \mathrm{p}=.005\right)$, los "horarios restringidos" ( $\left.\mathrm{Chi}^{2}=5.40 ; \mathrm{p}=.020\right)$ y la "falta de voluntad" (Chi² $\left.=8.26 ; \mathrm{p}=.004\right)$, que parece ser más importante en las 


\title{
MOTIVACIONES PARA EL EJERCICIO FÍSICO Y SU RELACIÓN CON LA SALUD MENTAL Y FÍSICA: UN ANÁLISIS DESDE EL GÉNERO
}

mujeres, si bien la primera fue la más frecuente en ambos géneros. Además, las personas que practican ejercicio físico muestran una mejor percepción de la salud física ( $t=7.87 ; p \leq .001)$ y mental $(t=2.31 ; p=.021)$. Se concluye que existen diferencias en la frecuencia y motivaciones para realizar ejercicio en función del género, relacionadas con los estereotipos de género. Además, las personas que realizan niveles mayores niveles de ejercicio físico perciben una mejor salud física y mental.

Palabras clave: ejercicio físico; género; motivaciones; percepción de salud física; percepción de salud mental

\begin{abstract}
Motivations for doing exercise and its association with mental and physical health: a gender

analysis. Physical exercise is a planned and structured physical activity with a final objective that constitutes a non-communicable diseases preventive tool and a physical and mental health protective factor. Likewise, the motivations for doing exercise are essential and it can be influenced by gender. The purpose of this study is to consider the differences about the motivations of doing exercise based on gender and to analyse the relationship between physical exercise and perceived physical and mental health. To achieve this objective, a sample of 600 young university students (50\% men and 50\% women), completed the online questionnaire about Lifestyle and Health (Giménez-García \& Ballester-Arnal, 2017). The analysis showed significant differences in the regular physical exercise between men (61.7\%) and women (45.3\%) $\left(\mathrm{Chi}^{2}=16.01 ; \mathrm{p} \leq .001\right)$. Moreover, gender differences in motivations for doing exercise were observed in all motivations, except for the motivation about "to be thinner" $\left(\mathrm{Chi}^{2}=1.00 ; \mathrm{p}=.317\right)$, being men who exceed women for all of them. In relation to the motivations for not doing exercise, there are differences for the motivations "lack of time" (Chi ${ }^{2}=7.72$; $\left.p=.005\right)$, "being short of time" $\left(\mathrm{Chi}^{2}=5.40 ; \mathrm{p}=.020\right)$ and "Lack of force of will" (Chi $\left.{ }^{2}=8.26 ; \mathrm{p}=.004\right)$, being more relevant for women. Moreover, people who practice exercise report better perceived physical ( $t=7.87 ; p \leq .001)$ and mental $(t=2.31 ; p=.021)$ health. Therefore, gender differences about frequency and motivations for doing exercise exist and are related to gender stereotypes. Additionally, people who do greater levels of physical exercise, have a better physical and mental health perception.
\end{abstract}

Keywords: physical exercise; gender; motivations; perceived physical health; perceived mental health

\section{INTRODUCCIÓN}

Diversos estudios reflejan el impacto beneficioso del ejercicio físico (en adelante, EF) sobre la salud y la percepción subjetiva que se tiene de ella, tanto en población joven como en personas más adultas, así como la contribución del EF a la disminución de la ansiedad y depresión, con un consecuente aumento de la calidad de vida (García-Molina, Baeza y Fernández, 2010; Olmedilla, Ortega y Candel, 2010; Stein, Molinero, Salguero, Corrêa y Márquez, 2014). La literatura muestra que los niveles de salud mental son mejores cuando se realiza ejercicio en niveles altos o moderados, además de reducir en un $56 \%$ el riesgo de padecer patologías mentales (RodríguezRomo, Barriopedro, Alonso-Salazar y Garrido-Muñoz, 2015). Asimismo, la práctica de EF se relaciona con una mejora de la condición física y de otros parámetros corporales que son indicativos de salud, como es el caso de la grasa corporal, previniendo el sobrepeso durante la etapa activa e incluso años más tarde (Ortega, Ruíz y Castillo, 2013). Por tanto, se podría afirmar que el ejercicio físico incrementa la salud, a la vez que previene las enfermedades no transmisibles (OMS, 2018). De esta forma, se apoyaría la tesis que ya defendía Wenzel (1983), desde hace décadas, al identificar el EF como uno de los 6 pilares del estilo de vida que proporciona mayores beneficios a la salud.

En este contexto, la población joven y adolescente parece presentar un perfil de riesgo. De hecho, algunos estudios indican cómo, entre un 60 y 80\% de adolescentes y jóvenes, reconocen ser sedentarias (Díaz-Cabrera, Carmona-Álamos y García-Tascón, 2017; OMS, 2018; Oviedo et al., 2013). Si bien distintas variables pueden incidir en la práctica de ejercicio físico, las motivaciones para realizarla cobrarían gran relevancia (MorenoMurcia, Martínez-Galindo y Alonso-Villodre, 2006; Rico-Díaz et al., 2019; Rodríguez-Romo et al., 2016). Desde 
Ios modelos cognitivos, la Teoría de la Acción Planificada (TAP; Ajzen, 1985) y la Teoría de la Autodeterminación (TAD; Deci y Ryan, 1985) enfatizan su importancia. Mientras que la TAP asume que las personas escogen racionalmente sus comportamientos, evaluando sus actitudes en relación con dichos comportamientos y el contexto, la TAD afirma que las personas basan sus intenciones hacia la realización de EF en los motivos generados por sus necesidades psicológicas, y estos se clasificarían como intrínsecos, extrínsecos o amotivacionales en función del grado de determinación. Tal y como muestra la literatura, ambas teorías se complementan para explicar la realización de ejercicio, puesto que cuando las motivaciones intrínsecas se acompañan de las actitudes idóneas, se traducen en dicha conducta (Hagger, Chatzisarantis y Biddle, 2002).

Con respecto a las diferencias de género, se registra que las mujeres realizan EF con una frecuencia más baja en comparación con los hombres, hecho que, según algunos estudios (Codina y Pestana, 2012; Oviedo et al., 2013), Ileva a plantear la existencia de estereotipos y roles de género que afectan a la práctica de EF. Estos vinculan el deporte con la masculinidad y no tanto con la feminidad, lo que favorece las actitudes negativas de muchas mujeres hacia el deporte. Asimismo, en lo referente al tipo de ejercicio practicado, las mujeres acostumbran a practicar deportes individuales y estéticos como el aerobic o la natación, mientras que los hombres realizan mayormente deportes colectivos y competitivos como el fútbol o básquet, elecciones que responden a los estereotipos creados (Moreno-Murcia et al., 2006). La literatura señala que el descenso del EF en mujeres, además de deberse a la masculinización del deporte, se relaciona con la menor disposición de tiempo por parte de las mujeres, ya que el EF ha de conciliarse con otros ámbitos como el trabajo, familia y/o ocio (Codina y Pestana, 2012; Hargreaves, 1993). Más allá de estos condicionantes, todavía falta evidencia sobre aspectos tan importantes como las posibles motivaciones diferenciales para hacer ejercicio, entre hombres y mujeres.

Con todo, dado el perfil sedentario de la población joven y las implicaciones que esto puede conllevar para su salud, parece necesario incrementar los esfuerzos de educación para la salud y así promover el EF en este segmento de la sociedad. Teniendo en cuenta la importancia de conocer más extensamente las motivaciones de la población diana para desarrollar estrategias eficaces, así como las lagunas existentes en la literatura, el presente trabajo se propone analizar las diferentes motivaciones para hacer ejercicio en función del género, así como observar la relación entre la realización de ejercicio físico y la percepción de salud física y mental.

\section{MÉTODO}

\section{Participantes}

La muestra está conformada por 600 personas, 300 mujeres (50\%) y 300 hombres (50\%), cursando estudios superiores. Del total de la muestra, el 52.8\% reconoce practicar ejercicio físico habitualmente. En cuanto a la salud física y mental, el $77.1 \%$ y el $78 \%$ considera que es buena o muy buena respectivamente, correspondiendo el porcentaje restante a los participantes que consideran que su salud física y/o mental es regular, mala o muy mala.

\section{Instrumentos}

El Cuestionario de Estilo de Vida y Salud para población joven (Giménez-García y BallesterArnal, 2017) evalúa factores de riesgo y protectores relacionados con la salud en población joven, a través de diferentes ámbitos como la alimentación, el consumo de sustancias, la actividad física o el uso de internet. Para el siguiente estudio, se ha utilizado la información de tres ítems relativos al ejercicio físico, concretamente, sobre la realización de ejercicio físico, las motivaciones para practicarlo y las barreras para hacerlo. La primera de estas variables fue medida a través de un formato de respuesta dicotómico y las dos restantes, a través de un formato de respuesta múltiple, en el que las personas podían elegir entre distintas motivaciones y barreras. Además, se analizaron dos ítems relativos a la salud física y la salud mental, los cuales se evaluaron a través de una escala tipo Likert (1=muy mala, 5=muy buena). 


\section{MOTIVACIONES PARA EL EJERCICIO FÍSICO Y SU RELACIÓN CON LA SALUD MENTAL Y FÍSICA: UN ANÁLISIS DESDE EL GÉNERO}

\section{Procedimiento}

Este trabajo se enmarca en un estudio más amplio que tiene como objetivo conocer los hábitos de salud y los estilos de vida de la población universitaria. Para ello, se difundió a través de redes sociales un mensaje en el que los participantes podían encontrar datos sobre el objetivo del estudio y la obligación de facilitar el consentimiento informado para participar. Asimismo, también se facilitaba información relativa a la garantía del anonimato y la voluntariedad, al formar parte de dicho estudio. Una vez dado el consentimiento, las personas podían acceder al cuestionario online. Para realizar el estudio se contó con la autorización de la Comisión Deontológica de la Universitat Jaume I.

\section{Análisis de datos}

Los datos recabados y posteriormente registrados fueron analizados con el programa estadístico IBM SPSS Statistics 20. Por un lado, la caracterización de la muestra fue estudiada a través del cálculo de estadísticos descriptivos. Por otro lado, Ios análisis diferenciales de las motivaciones y barreras según género se realizaron con la prueba Chi cuadrado, mientras el análisis para valorar si existían diferencias entre las personas que realizaban o no actividad física respecto a la percepción de la salud, se realizó con la prueba t.

\section{RESULTADOS}

En primera instancia se observa, en la Figura 1, que las motivaciones más frecuentes para realizar ejercicio son las correspondientes a "Tener mejor salud física" y "Me relaja", seguido de "Por diversión" y "Tener mejor aspecto físico", mientras que aquellas menos frecuentes son las referidas a "Conocer otras personas" y "Estar más delgado/a". Si bien esta última, la reporta el $13.8 \%$ de la muestra.

Figura 1. Distribución de las motivaciones para realizar EF en la muestra total ( $N=600)$



Por otro lado, en cuanto a los motivos para no hacer ejercicio, destacan más frecuentemente la "Falta de tiempo", "Falta de voluntad o pereza" y "Mis horarios no me lo permiten". Por el contrario, los obstáculos menos frecuentes son "Me lo impiden mis problemas de salud", "Porque me aburre" y "Porque no me gusta" (Ver Figura 2). 
Figura 2. Distribución de las barreras para realizar EF en la muestra total ( $N=600)$

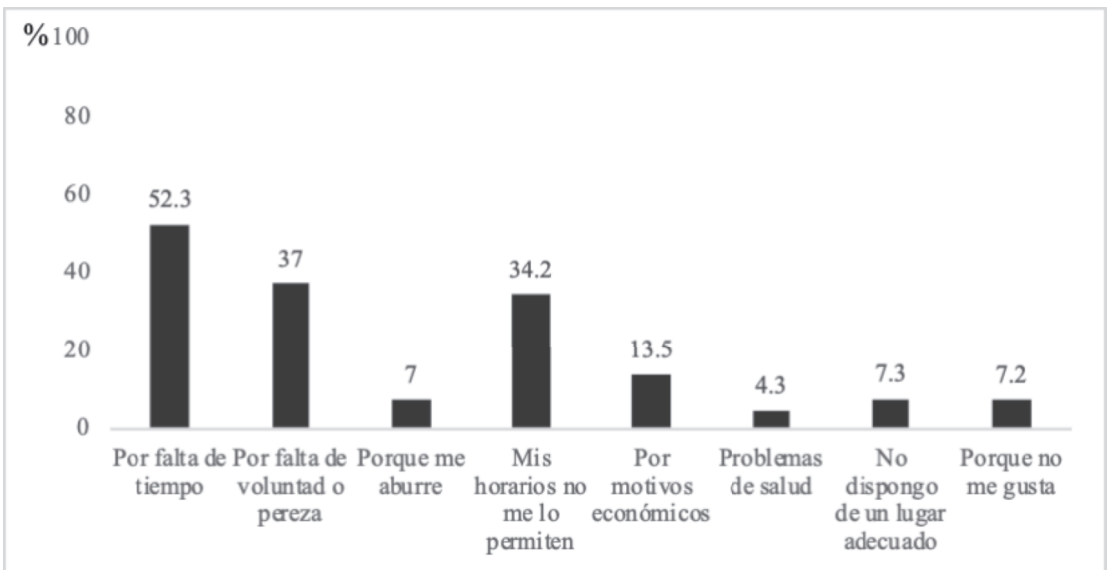

Por lo que respecta a la realización de ejercicio en función del género (ver Figura 3), existen diferencias estadísticamente significativas ( $\mathrm{Chi}^{2}=16.01 ; \mathrm{p}=\leq .001$ ), siendo los porcentajes globales de realización de EF mayores en hombres que en mujeres.

Figura 3. Distribución de la realización de ejercicio físico según género

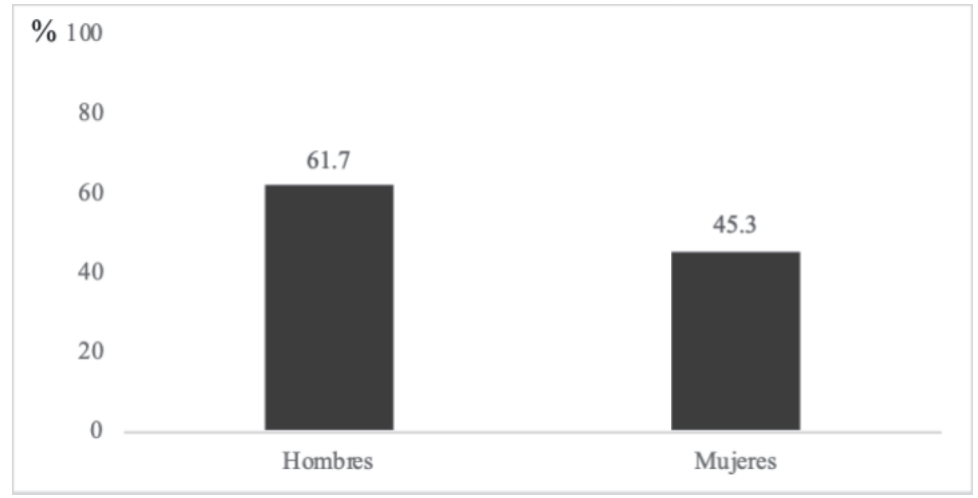

Por lo que respecta a las motivaciones para realizar ejercicio, entre mujeres y hombres (ver Tabla 1), los resultados evidenciaron diferencias estadísticamente significativas en todas las motivaciones, excepto en el motivo "Estoy más delgado". Además, en todos los casos son más frecuentes en los hombres. La motivación para realizar ejercicio más frecuente en las mujeres es la relativa a "Me relaja", seguida de "Mejorar la salud física" y "Mejorar el aspecto físico". En el caso de los hombres, se corresponde con tener "Mejor salud física", "Me relaja" y "Diversión". 
Tabla 1

Análisis diferencial de las motivaciones para realizar ejercicio según género

\begin{tabular}{lcccc}
\hline & $\begin{array}{c}\text { Hombre } \\
(\mathrm{n}=300) \\
(\%)\end{array}$ & $\begin{array}{c}\text { Mujer } \\
(\mathrm{n}=300) \\
(\%)\end{array}$ & $\mathrm{Chi}^{2}$ & $p$ \\
\hline Diversión & 44.4 & 27.2 & 19.15 & $\leq .001$ \\
Me relaja & 47.1 & 35.9 & 7.67 & .006 \\
Estoy más fuerte & 41.4 & 18.5 & 37.12 & $\leq .001$ \\
Estoy más ágil & 36.6 & 20.1 & 19.82 & $\leq .001$ \\
Mejor salud física & 48.8 & 34.2 & 12.99 & $\leq .001$ \\
Mejor aspecto físico & 40.7 & 28.2 & 10.24 & .001 \\
Estoy más delgado & 15.3 & 12.4 & 1.00 & .317 \\
Conocer otras personas & 18 & 6.4 & 18.66 & $\leq .001$ \\
\hline
\end{tabular}

En cuanto a las barreras percibidas (ver tabla 2), en ambos géneros, los inconvenientes percibidos más frecuentes para realizar EF son "Falta de tiempo", "Falta de voluntad y pereza" y "Mis horarios no me lo permiten", además de mostrar diferencias estadísticamente significativas en ambos géneros. Con excepción de la barrera "No dispongo de un lugar adecuado", las dificultades percibidas son, en todos los casos, más frecuentes en las mujeres.

Tabla 2

Análisis diferencial de las barreras para realizar ejercicio según género

\begin{tabular}{lcccc}
\hline & $\begin{array}{c}\text { Hombre } \\
(\mathrm{n}=300) \\
(\%)\end{array}$ & $\begin{array}{c}\text { Mujer } \\
(\mathrm{n}=300) \\
(\%)\end{array}$ & $\mathrm{Chi}^{2}$ & $p$ \\
\hline Falta de tiempo & 46.7 & 58 & 7.72 & .005 \\
Falta de voluntad o pereza & 31.3 & 42.7 & 8.26 & .004 \\
Me aburre & 6 & 8 & 0.92 & .337 \\
Mis horarios no me lo permiten & 29.7 & 38.7 & 5.40 & .020 \\
Motivos económicos & 11 & 16 & 3.21 & .073 \\
Problemas de salud & 3.3 & 5.3 & 1.44 & .229 \\
No dispongo de lugar adecuado & 7.7 & 7 & 0.09 & .754 \\
No me gusta & 5.3 & 9 & 3.03 & .082 \\
\hline
\end{tabular}


En última instancia, se analizaron las diferencias en la percepción de la salud física y mental según la realización o no de actividad física. Aquellas personas que realizan ejercicio físico parecen percibir mejor su salud física y mental que las que no lo practican (ver Tabla 3).

Tabla 3.

Diferencias en percepción de salud física y mental según se práctica o no ejercicio físico

\begin{tabular}{lccccc}
\hline & \multicolumn{2}{c}{ Sí realizan EF } & \multicolumn{2}{c}{ No realizan EF } & $\mathrm{t}(\mathrm{p})$ \\
\cline { 2 - 6 } & $\mathrm{M}$ & $\mathrm{DT}$ & $\mathrm{M}$ & $\mathrm{DT}$ & \\
Salud física & 4.20 & 0.59 & 3.81 & 0.60 & $7.87(.000)$ \\
Salud mental & 4.06 & 0.77 & 3.90 & 0.88 & $2.31(.021)$ \\
\hline
\end{tabular}

\section{DISCUSIÓN Y CONCLUSIONES}

El objetivo propuesto para este estudio fue el de analizar las diferencias entre hombres y mujeres por lo que respecta a las motivaciones y barreras para hacer deporte, así como la percepción de salud física y mental, según la realización o no de ejercicio. En líneas generales, tal y como muestran otros estudios (Díaz-Cabrera et al., 2017; OMS, 2018; Oviedo et al., 2013), los jóvenes reportan niveles importantes de sedentarismo.

En cuanto a las motivaciones más prevalentes para hacer EF entre la población joven participante, se observó que coinciden con aquellas relacionadas con la salud, la relajación y el disfrute, mientras que las menos frecuentes son las relacionadas con las relaciones sociales, siendo resultados similares a los de otros estudios (Ahedo y Macua, 2016; Díaz-Cabrera et al., 2017; Rico-Díaz et al., 2019; Rodríguez-Romo et al., 2016). Ahora bien, a diferencia de otros estudios (Rodríguez-Romo et al., 2016), en estos hallazgos se muestra más relevancia de la apariencia física, puesto que la búsqueda de una mejor apariencia física se reporta como una motivación frecuente. Esto sugiere que hay un elevado deseo entre los participantes de tener un cuerpo atractivo, en base al ideal corporal establecido socialmente, y el deporte se concebiría como una forma de alcanzarlo (Capdevila, Niñerola y Pintanell, 2004)

Por otro lado, en cuanto a las barreras percibidas, destacan aquellas relacionadas con la falta de tiempo y Ios horarios restringidos. Este hecho va en línea con un estudio llevado a cabo por Londoño, Cifre y Rosel (2016), afirmando que las jornadas largas y las cargas de trabajo elevadas limitan el tiempo libre, disminuyendo también la probabilidad de hacer EF. De esta forma, cabría enfatizar las propuestas que destacan la importancia de crear una oferta de deportes que sea compatible y adaptada a los diferentes horarios, así como facilitar el acceso al mismo y la conciliación con el resto de las tareas (Sevil, Práxedes, Zaragoza, Del Villar y García-González, 2015; Rico-Díaz, et al., 2019). Según el estudio de Abu-Omar y Rutten (2008), la actividad física que se realiza en el tiempo libre, la cual incluye el EF, sería la que tiene un mayor un impacto positivo sobre la salud. Otro inconveniente percibido para la realización de ejercicio físico es la falta de voluntad, hecho que reafirma la importancia de encontrar formas de incrementar la motivación de los jóvenes (Moreno-Murcia et al, 2006; Rico-Díaz et al., 2019; Rodríguez-Romo et al., 2016)

Por lo que respecta al análisis en función del género, al igual que en pasados estudios (Herrera-Gutiérrez, Brocal-Pérez, Sánchez y Rodríguez, 2012; Rico-Díaz, et al., 2019), los hombres reportan más ejercicio físico que las mujeres. En cuanto a las motivaciones para realizar ejercicio físico observadas en este estudio, si bien todas parecen ser más prevalentes entre los hombres, destacarían aquellas relacionadas con estar más fuerte y ágil, además de informar una mayor asociación entre la práctica deportiva y la diversión. Estos resultados señalan una mayor motivación para la realización de ejercicio físico por parte de los hombres que, tal y como señala la literatura anterior (Codina y Pestana, 2012; Moreno-Murcia et al., 2006), podría relacionarse con los estereotipos de género y, en concreto, con los atributos que caracterizan la masculinidad. En las mujeres, por el contrario, parece que tener un cuerpo más bonito motiva más que la diversión que se pueda alcanzar con el ejercicio. 


\section{MOTIVACIONES PARA EL EJERCICIO FÍSICO Y SU RELACIÓN CON LA SALUD MENTAL Y FÍSICA: UN ANÁLISIS DESDE EL GÉNERO}

Por lo que respecta a las barreras percibidas para la realización de EF en función del género, los resultados muestran diferencias estadísticas en la pereza o falta de voluntad, así como en los motivos referidos a la falta de tiempo y los horarios restringidos, siendo las mujeres las que más afirmaron percibirlas. El informe de estas últimas barreras iría en la línea de trabajos que afirman que las mujeres practican menos deporte a causa de una mayor dificultad para conciliar el trabajo o estudios y la familia y ocio, respecto a los hombres (Codina y Pestana, 2012; Hargreaves,1993).

Finalmente, los resultados mostraron una mejor percepción de salud física y mental entre las personas que practican ejercicio físico. De esta forma, se apoyarían pasados estudios que, además, mostraron cómo una mayor práctica de EF se relaciona a su vez con una mayor adherencia a la dieta mediterránea y un menor consumo de alcohol, factores que a su vez contribuyen a la salud de los individuos (Moral-García et. al., 2018). Asimismo, diversos estudios respaldan que la práctica de EF contribuye a una mejora de la estabilidad psicológica y de la reducción de la ansiedad y depresión (Herrera-Gutiérrez et al., 2012; Olmedilla et al., 2010).

Estos resultados cabría tenerlos en cuenta a la vista de algunas limitaciones. Sería interesante replicar el estudio con una muestra mayor y de distinto perfil sociodemográfico, para así garantizar la generalización de los resultados del estudio. Al mismo tiempo, se podrían incluir medidas más objetivas que complementen los resultados del autoinforme utilizado que recoge la percepción subjetiva de la práctica del ejercicio físico, así como de la salud mental y física.

En definitiva, este estudio se suma a aquellos que apuntan a la necesidad de promover una mayor práctica de actividad física entre la población joven, teniendo en cuenta las motivaciones para realizar EF. Si bien algunas de ellas resultarían adaptativas, otras, podrían basarse en elementos menos saludables que fomentaran un uso indebido del ejercicio u otras conductas de riesgo para la salud, relacionadas con el culto al cuerpo o los estereotipos de género. Además, dadas las diferencias encontradas, también parece evidente la necesidad de que los esfuerzos preventivos se aborden de manera diferencial en función del género.

\section{REFERENCIAS BIBLIOGRÁFICAS}

Abu-Omar, K. y Rutten, A. (2008). Relation of leisure time, occupational, domestic, and commuting physical activity to health indicators in Europe. Preventive Medicine, 47(3), 319-323. doi:10.1016/j.ypmed.2008.03.012

Ahedo, R. y Macua, A. (2016). Características de las prácticas de ocio físico-deportivas significativas de los jóvenes españoles. Revista de Psicología del Deporte, 25(2), 67-72 Recuperado de: https://www.rpdonline.com/article/view/v25-n4-ahedo-macua/Ahedo_Macua

Ajzen, I. (1985). From intentions to actions: Atheory of planned behaviour. En: J. Kuhl \& J. Beckmann (Eds.), Action-control: From cognition to behaviour (pp. 11-39). Heidelberg: Springer.

Capdevila, L., Niñerola, J. y Pintanell, M. (2004). Motivacion y actividad fisica: el autoinforme de motivos para la practica de ejercicio fisico (AMPEF). Revista de Psicología del Deporte, 13(1), 55-74. Recuperado de: https://www.rpd-online.com/article/view/261/capdevila\%20131

Codina, N. y Pestana, J.V. (2012). Estudio de la relación del entorno psicosocial en la practica deportiva de la mujer. Revista de Psicología del Deporte, 21(2), 243-251. Recuperado de: http://diposit.ub.edu/dspace/bitstream/2445/36361/1/604773.pdf

Deci, E. L. y Ryan, R. M. (1985). Intrinsic motivation and self-determination in human behaviour. New York: Plenum Press.

Díaz-Cabrera, L.A., Carmona-Álamos, L. y García-Tascón, M. (2017). Análisis de la práctica deportiva de alumnos de la universidad Pablo de Olavide, Sevilla (España) en función del género. PODIUM Sport, Leisure and Tourism Review, 6(3). D0I: 10.5585/podium.v6i3.230

García-Molina, A. V. A., Baeza, A. \& Fernández, M. (2010). Beneficios de la actividad física en personas mayores. Revista Internacional de Medicina y Ciencias de la Actividad Física y del Deporte, 10 (40), 556-576. Recuperado de: Http://cdeporte.rediris.es/revista/revista40/artbeneficios181.htm 
Giménez-García, C. y Ballester-Arnal, R. (2017). Cuestionario de Estilo de Vida y Salud para Población Joven. Manuscrito no publicado, Universitat Jaume I de Castellón, España.

Hagger, M. S., Chatzisarantis, N. L. y Biddle, S. J. (2002). The influence of autonomous and controlling motives on physical activity intentions within the Theory of Planned Behaviour. British Journal of Health Psychology, 7(3), 283-297. DOI: 10.1348/135910702760213689

Hargreaves, J. (1993). Problemas y promesa en el ocio y los deportes femeninos. En Brohm J. y Bourdieu, P. (Ed.) Materiales de sociología del deporte (pp. 109-132). Madrid, España: La Piqueta.

Herrera-Gutiérrez, E., Brocal-Pérez, D., Sánchez, D. J. y Rodríguez, J. M. (2012). Relación entre actividad física, depresión y ansiedad en adolescentes. Cuadernos de Psicología del Deporte, 12(2), 31-38. Recuperado de: https://www.redalyc.org/pdf/2270/227028254005.pdf

Londoño, M.E., Cifre, E. y Rosel J. (2016). Demandas-Control y Salud: El Rol Mediador del Tiempo Libre. Revista Interamericana de Psicologia Ocupacional, 34(1), 22-40. D0I:10.21772/ripo.v34n1a02

Moral-García, J.E., Agraso-López, A.D., Pérez-Soto, J.J., Rosa-Guillamón, A., Tárraga-Marcos, M.L., García Cantó, E. y Tárraga López, P.J. (2019). Práctica de actividad física según adherencia a la dieta mediterránea, consumo de alcohol y motivación en adolescentes. Nutrición Hospitalaria, 36(2), 420-427. D0I: http://dx.doi.org/10.20960/nh.2181

Moreno-Murcia, J. A., Martínez-Galindo, C. y Alonso-Villodre, N. (2006). Actitudes hacia la práctica físico-deportiva según el sexo del practicante. Revista Internacional de Ciencias del Deporte, 3(2), 20-43. Recuperado de: http://www.cafyd.com/REVISTA/art2n3a06.pdf

Olmedilla, A., Ortega, E. y Candel, N. (2010). Ansiedad, depresión y práctica del ejercicio físico en estudiantes universitarias. Apunts Medicina de l'Esport, 45 (167), 175-180. Recuperado de: https://www.apunts.org/enpdf-X0213371710545625

Organización Mundial de la Salud (2018) Actividad Física. Ginebra, Suiza. Recuperado de: https://www.who.int/es/news-room/fact-sheets/detail/physical-activity

Ortega, F.B., Ruíz, J.R. y Castillo, M.J. (2013) Actividad física, condición física y sobrepeso en niños y adolescentes: evidencia procedente de estudios epidemiológicos. Endocrinología y Nutrición, 60(8), 458-469. D0I: 10.1016/j.endonu.2012.10.006

Oviedo, G., Sánchez, J., Castro, R., Calvo, M., Sevilla, J. C., Iglesias A. y Guerra, M. (2013). Niveles de actividad física en población adolescente: estudio de caso. Retos. Nuevas tendencias en Educación Física, Deporte y Recreación, 23, 43-47. Recuperado de: https://dialnet.unirioja.es/servlet/articulo?codigo=4135239

Rico-Díaz, J., Arce-Fernández, C., Padrón-Cabo, A., Peixoto-Pino, L. y Abelairas-Gómez, C. (2019). Motivaciones y hábitos de actividad física en alumnos universitarios. Retos, 36, 446-453. Recuperado de: https://recyt.fecyt.es/index.php/retos/article/view/69906/43947

Rodríguez-Romo, G., Barriopedro, M., Alonso-Salazar, P.J. y Garrido-Muñoz, M. (2015). Relaciones entre Actividad Física y Salud Mental en la Población Adulta de Madrid. Revista de Psicología del Deporte, 24(2), 233-239. Recuperado de: https://www.rpd-online.com/article/view/v24-n2-rodriguez-romo-barriopedroetal/Rodriguez_Romo_Barriopedroetal

Rodríguez-Romo, G., Macias-Pla, R, Garrido-Muñoz, M, Tejero-Gonzalez, C.M. y López-Adan, E. (2016) Motivos para la práctica de actividad física durante el tiempo libre y su relación con el cumplimento de las recomendaciones. Cuadernos de Psicología del Deporte, 18(1), 183-194. Recuperado de: https://revistas.um.es/cpd/article/view/274141/225181

Sevil, J., Práxedes, A., Zaragoza, J., Del Villar, F. y García, L. (2015). Barreras percibidas para la práctica de actividad física en estudiantes universitarios. Diferencias por género y niveles de actividad física. Universitas Psichologica, 16(4) doi: 10.11144/Javeriana.upsy16-4.bppa

Stein, A. C., Molinero, 0., Salguero, A., Corrêa, M. C. R. y Márquez, S. (2014). Actividad física y salud percibida en pacientes con enfermedad coronaria. Cuadernos de Psicología del Deporte, 14(1), 109-116. Recuperado 
MOTIVACIONES PARA EL EJERCICIO FÍSICO Y SU RELACIÓN CON LA SALUD MENTAL Y FÍSICA:

UN ANÁLISIS DESDE EL GÉNERO

de: http://scielo.isciii.es/pdf/cpd/v14n1/art12.pdf

Wenzel, E. (1983). Lifestyles and living conditions and their impact on health: a report of the meeting. European Monographs in Health Education Research, 5, 1-18. 